\title{
Spin Wave Specific Heat in Quasiperiodic Fibonacci Structures
}

\author{
C.G. Bezerra ${ }^{a}$, E.L. Albuquerque ${ }^{\mathrm{b}}$ *, A.M. Mariz ${ }^{\mathrm{b}}$, \\ L.R. da Silva ${ }^{\mathrm{b}, \mathrm{c}}$, and C. Tsallis ${ }^{\mathrm{d}}$
}

November 13, 2018

(a) Department of Physics and Astronomy, University of Western Ontario, London-Ontario N6A3K7, Canada

(b) Departamento de Física, Universidade Federal do Rio Grande do Norte, Natal-RN 59072-970, Brazil

(c) Department of Physics, Boston University, Boston-MA 02215, USA

(d) Centro Brasileiro de Pesquisas Físicas, Rua Xavier Sigaud 150, 22290-

180 Rio de Janeiro-RJ, Brazil

\begin{abstract}
The energy spectra of a variety of collective modes on quasiperiodic structures exhibit a complex fractal profile. Among the modes that have attracted particular attention in this context, are the spin wave spectra of quasiperiodic magnetic multilayers that obey a substitutional sequence of the Fibonacci type. They are described within the framework of the Heisenberg theory. In order to have a deep insight on the relevant thermodynamical implications of the above mentioned energy spectra's fractal profile, we have performed analytical and numerical calculations of the spin wave specific heat associated with successive hierarchical sequences of the Fibonacci quasiperiodic structures. The spectra show interesting oscillatory behavior in the low-temperature region, which can be traced back to the spin wave's self-similar energy spectrum.
\end{abstract}

PACS: 05.20-y; 61.43.Hv; 61.44.Br; 75.30.Ds

Keywords: Quasi-Crystals; Spin waves; Fractal behavior; Thermodynamical properties

${ }^{*}$ Corresponding author, e-mail: ela@dfte.ufrn.br 


\section{Introduction}

Since the discovery of the icosahedral phase in Al-Mn alloys by means of $\mathrm{X}$-ray spectroscopy [1], the quasicrystaline systems have been extensively studied (for a revision see [2]). In particular, the physical properties of the so-called quasiperiodic structures have attracted a lot of attention from both theoretical and experimental point of view. Although the term quasicrystal is more appropriate for natural compounds or artificial alloys, in one dimension there is no difference between this case and the quasiperiodic structure formed by the incommensurate arrangement of periodic unit cells. Due to this motivation, Merlin and collaborators, using the molecular beam epitaxy (MBE) technique, grew in 1985 the first quasiperiodic superlattice following the Fibonacci sequence [3]. After that pioneer work, other quasiperiodic structures were experimentally realized [4].

¿From a theoretical point of view, a number of physical properties has been studied in quasiperiodic structures. Among them we can cite the energy spectra of polaritons [5], phonons [6], electrons [7] and spin waves [8, 9], as well as the magnetoresistance and magnetization curves of quasiperiodic thin films [10]. A quite interesting feature, common to all of these systems, is a self-similar pattern of their spectra. In fact, the energy spectra of the above referred particles and systems are highly fragmented and tend to Cantor sets in the thermodynamic limit. The origin of this fractality can be attributed to the long range order induced by the non-usual hierarchical structure of the quasiperiodic sequences used in the construction of the system.

A very interesting question aroused in the last few years: what are the consequences of a fractal energy spectra on the behavior of the thermodynamic properties of quasiperiodic systems? In a recent work, Tsallis and collaborators [11] studied the specific heat properties of a fractal energy spectra generated by the geometrical triadic Cantor set. They have found, as the main result of the paper, that the specific heat presents oscillations around the fractal dimension of the spectra. In addition, a non-uniform convergence between the so-called banded and discrete models was observed. Their results were extended by Vallejos et al [12, 13] for the two-scale Cantor set case. For this more general situation, the specific heat also exhibits log-periodic oscillations around the fractal dimension. Later Carpena et al [14], using the properties of a multifractal spectra showed under what conditions the oscillatory regime disappears. Finally, Curado and Rego-Monteiro [15] examined the thermodynamic properties of a solid exhibiting the energy spectrum given by a logistic map. 
The aim of this work is to push a little bit more the understanding of the thermodynamic properties of quasicrystals, analyzing the specific heat of a real fractal spectra of spin waves in quasiperiodic Fibonacci magnetic superlattices. We consider that the building blocks used here to set up the quasiperiodic structures are ferromagnetic materials, whose dynamics are described by the Heisenberg Hamiltonian. Our main concern is to emphasize the differences between the specific heat profile obtained by using a real spin wave's multifractal energy spectra for a given in-plane wavevector, (with, by instance, their proper scaling laws), as discussed in Ref. 8, and an idealized Cantor set, considered in previous works. Throughout the paper we use the classical Maxwell-Boltzmann statistics.

This paper is organized as follows: In section II we discuss the theoretical method used to obtain the spin waves dispersion relation (their energy spectra), and the rules used to build up the Fibonacci superlattice. Section III is devoted to the specific heat theory of a general scaled energy band spectra. The application of this theory to the Fibonacci quasiperiodic structure is then made, and the main features of the spectra obtained are discussed. Finally, section IV presents the conclusions of this work.

\section{Physical model}

In this section we briefly describe the physical model for the quasiperiodic magnetic superlattices. A detailed description can be found in Ref. [8]. We consider superlattices in which $n_{A}$ layers of material $A$ (building block $A$ ) alternate with $n_{B}$ layers of material $B$ (building block $B$ ). Both materials are taken to be simple cubic spin- $S$ Heisenberg ferromagnets having exchange constants $J_{A}$ and $J_{B}$, respectively, and lattice constant $a$. At the interface $A / B$, the exchange constant is suppose to be equal to $I$. The Heisenberg Hamiltonian for each component is:

$$
\mathcal{H}=(-1 / 2) \sum_{i, j} J_{i j} \vec{S}_{i} \cdot \vec{S}_{j}-g \mu_{B} H_{0} \sum_{i} S_{i}^{z}
$$

Here the sum in the first term is over sites $i$ and nearest neighbors (n.n.) $j$, and $H_{0}$ is a static external magnetic field pointing in the $z$-direction .

The spin wave dispersion relation in a superlattice can be found by solving the equations of motion for the operator $S_{i}^{+}=S_{i}^{x}+i S_{i}^{y}$, i.e: 


$$
\hbar \frac{\partial}{\partial t} S_{i}^{+}=g \mu_{B} H_{0} S_{i}^{+}+<S^{z}>\sum_{n . n .} J_{A}\left(S_{i}^{+}-S_{j}^{+}\right),
$$

where $\left\langle S^{z}\right\rangle$ is the random phase approximation for the $z$-component of the spin operator. The solution of (2), for material $A$, is:

$$
S_{i}^{+}=\left[A_{l} \exp \left(i \vec{k}_{A} \cdot \vec{r}_{A}\right)+B_{l} \exp \left(-i \vec{k}_{A} \cdot \vec{r}_{A}\right)\right](\exp -i \omega t)
$$

with similar expression for material $B$. These solutions are linked together using the equation of motion (2) at the boundaries of the $n$-th unit cell, which can be written in a matricial form. Using the translational invariance property of the excitations, through Bloch's theorem, the spin wave dispersion relation follows as:

$$
\cos (Q D)=(1 / 2) \operatorname{Tr}[\mathbf{T}]
$$

Here $Q$ is the Bloch'wavevector of the collective mode, $D$ is the size of the superlattice's unit cell, and $\mathbf{T}$ is a transfer matrix which relates the coefficients of the $(l+1)$ th cell to the coefficients of the preceding one.

A Fibonacci superlattice can be grown experimentally by juxtaposing the two building blocks $A$ and $B$ in such way that the $n t h$-generation of the superlattice $S_{n}$ is given iteratively by the rule $S_{n}=S_{n-1} S_{n-2}$, for $n \geq 2$, with $S_{0}=B$ and $S_{1}=A$. It is also invariant under the transformations $A \rightarrow A B$ and $B \rightarrow A$. The Fibonacci generations are,

$$
S_{0}=[B], S_{1}=[A], S_{2}=[A B], S_{3}=[A B A] \text {, etc. }
$$

The number of building blocks increases according to the Fibonacci number, $F_{n}=F_{n-1}+F_{n-2}\left(\right.$ with $\left.F_{0}=F_{1}=1\right)$, and the ratio between the number of the building blocks $A$ and the number of building blocks $B$, when $n>>1$, in the sequence tends to $\tau=(1 / 2)(1+\sqrt{5})$, an irrational number known as the golden mean.

It can be shown that the transfer matrix for the $n t h$ generation of a Fibonacci superlattice can be obtained by a simple recurrence relation given by [8],

$$
T_{s_{n}}=T_{s_{n-2}} \cdot T_{s_{n-1}}, \quad n \geq 2 .
$$

Therefore, from the knowledge of the transfer matrices $T_{S_{0}}$ and $T_{S_{1}}$, we can determine the transfer matrix for any generation and consequently the dispersion relation. Note that the matrix $T_{S_{2}}$ recovers the periodic case. 


\section{$3 \quad$ Specific heat Spectra}

The spin wave fractal spectra for the Fibonacci superlattices is depicted in Fig. 1, for a fixed value of the in-plane dimensionless wavevector $k_{x} a$. From there we can see the forbidden and allowed energies of the spin wave spectra against the Fibonacci's generation number $n$, up to their 8 th generation, which corresponds an unit cell with $21 A$ 's and $13 B$ 's building blocks. The number of allowed bands is equal to three times the Fibonacci number $F_{n}$, of the correspondent generation. Notice that, as expected, for large $n$ the allowed band regions get narrower and narrower and they have a typical Cantor set structure.

We address now to the specific heat of the spectra indicated in Fig. 1. The description below is general and can be applied to any banded spectrum. In Fig. 1 each spectrum, for a fixed generation number $n$, has $m$ allowed continuous bands. We consider, without loss of generality, the level density within each band constant. The partition function for the $n t h$ generation is then given by:

$$
Z_{n}=\int_{0}^{\infty} \rho(\epsilon) e^{-\beta \epsilon} d \epsilon
$$

Here $\beta=1 / T$ (by choosing the Boltzmann's constant $k_{B}=1$ ), and we take the density of states $\rho(\epsilon)=1$. After a straightforward calculation we can write $Z_{n}$ as,

$$
Z_{n}=\frac{1}{\beta} \sum_{i=1,3, \ldots}^{2 m-1} e^{-\beta \epsilon_{i}}\left[1-e^{-\beta \Delta_{i}}\right] .
$$

Here the subscript $n$ is the generation number, $m$ is the number of allowed bands and $\Delta_{i}=\epsilon_{i+1}-\epsilon_{i}$ is the difference between the top and bottom energy levels of each band.

The specific heat is then given by,

$$
C_{n}(T)=\frac{\partial}{\partial T}\left[T^{2} \frac{\partial \ln Z_{n}}{\partial T}\right],
$$

which can be written as,

$$
C_{n}(T)=1+\frac{\beta f_{n}}{Z_{n}}-\frac{g_{n}^{2}}{Z_{n}^{2}},
$$


with,

$$
f_{n}=\sum_{i=1,3, \ldots}^{2 m-1}\left[\epsilon_{i}^{2} e^{-\beta \epsilon_{i}}-\epsilon_{i+1}^{2} e^{-\beta \epsilon_{i+1}}\right] .
$$

and,

$$
g_{n}=\sum_{i=1,3, \ldots}^{2 m-1}\left[\epsilon_{i} e^{-\beta \epsilon_{i}}-\epsilon_{i+1} e^{-\beta \epsilon_{i+1}}\right] .
$$

Therefore, once we know the energy spectra of the spin wave which propagates in a given sequence's generation of a quasiperiodic structure, we can determine the associated specific heat's spectra by using (10).

Fig. 2 shows the spin wave specific heat spectra of the Fibonacci superlattices, for the in-plane wavevector $k_{x} a=2.0$, as a function of the temperature. For the high temperature limit $(T \rightarrow \infty)$, the specific heat for all generation numbers converges and decays as $T^{-2}$, for arbitrary $n$, in agreement with the triadic case. This is a consequence of the existence of a maximum energy value in the spectrum (once the spectrum is bounded). As the temperature decreases, the specific heat increases up to a maximum value. The corresponding temperature for this maximum value depends on the Fibonacci generation number $n$, although one can see a clear tendency for a common temperature value as $n$ increases. After the maximum value, the specific heat falls into the low temperature region. In this region it starts to present non-harmonic small oscillation behavior, as shown in the inset of Fig. 2. These oscillations, are not around a specific fractal dimension of the quasiperiodic structure, as in the idealized triadic Cantor set! Besides, it cannot be considered also an approximation of the idealized oscillations found in the triadic Cantor set. Their profiles define clearly two classes of oscillations, one for the even and the other for the odd generation numbers of the sequence, the amplitude of the odd oscillations being bigger than the amplitude of the even one. These behaviors are better illustrated in Fig. 3 , where are depicted $\log$ - $\log$ plots of the specific heat against the temperature for several generation numbers. Of course the number of oscillations observed in the specific heat spectra is related to the hierarchical generation number $n$ (more oscillations appear as $n$ increases). Another behavior of these oscillations can be seen in Fig. 4, where we have plotted the specific heat against $\log T$. We can note a well defined period in the oscillations, which means that the specific heat is a log-periodic function of the temperature. The curves resemble the triadic case, with a mean value $d$, around it $C(T)$ oscillates log-periodically, although, as in the log-log plot, this value is 
not related with the fractal dimension of the Fibonacci quasiperiodic structure. Besides, the mean value is different for the even and odd Fibonacci's generation numbers.

\section{Conclusions}

In this paper we have studied the spin wave specific heat, for fixed values of the in-plane dimensionless wave vector $k_{x} a$, for the Fibonacci magnetic superlattices. We have shown, as a common aspect of the model employed here, that the specific heat tends to zero in the high temperature limit $(T \rightarrow$

$\infty)$, as $T^{-2}$, no matter its generation number. This asymptotic behavior is mainly due to the fact that we have considered our system bounded. It also presents a maximum whose corresponding temperature tends to a fixed value as the generation number increases. Finally, in the low temperature region, a small oscillations arises as an indication that the specific heat is a log-periodic function of the temperature. These oscillations can be defined as the signature of the quasiperiodic system, and has no counterpart in the idealized case.

It would be of interest to have experimental data to test our predicted theoretical results presented here. However, most of the experimental studies of magnetic multilayers to date, have been done for transition metals [16, 17]. Further experimental studies carried out for multilayers structures of magnetic insulator or magnetic semiconductor materials, that would be better described by the Heisenberg model employed in this paper, are welcome. Suitable experimental technique to probe the spin wave spectra in the quasi-periodic structure discussed here is the inelastic light scattering spectroscopy of Raman and Brillouin type. However, the spectra can be obtained only for a given in-plane wavevector $k_{x}$, which defines the incident angle $\theta$ of the light through the relation $\sin ^{-1} \theta=k_{x} \lambda / 4 \pi, \lambda$ being the the laser wavelength 18, 19]. This fact adds an extra complication to the experimental specific heat measurements, but we hope that the experimentalists can be encouraged to overcome it. Techniques involving magnetic resonance (for example, ferromagnetic resonance, standing spin-wave resonant etc.) can also be used, and indeed they were previously been successfully applied to surface and bulk spin waves in various magnetic microstructures (for a good account of these techniques see 201).

Acknowledgments: One of us (ELA) thanks the hospitality of the Center 
for Polymer Studies, Boston University-USA, where part of this work was done. We thank also fruitful discussions with Profs. H.E. Stanley and S.V. Buldyrev, as well as financial support from CNPq (Brazilian Agency) and PRONEX. 


\section{References}

[1] D. Shechtman, I. Blench, D. Gratias and J.W. Cahn, Phys. Rev. Lett. 53, 1951 (1984).

[2] P.A. Lee and T.V. Ramakrishnan, Rev. Mod. Phys. 57, 287 (1985); P.J. Steinhardt and S. Ostlund, The Physics of Quasicrystals, World Scientific, Singapore, 1987; D. Di Vicenzo and P.J. Steinhardt, Quasicrystals: The State of The Art, World Scientific, Singapore, 1991; C. Janot, Quasicrystals: A Primer, Oxford University Press, Oxford, 1993; M. Senechal, Quasicrystals and Geometry, Cambridge University Press, Cambridge, 1995.

[3] R. Merlin, K. Bajema, R. Clarke, F.-Y. Juang, and P.K. Bhattacharya, Phys. Rev. Lett 55, 1768 (1985).

[4] J. Todd, R. Merlin, R. Clarke, K.M. Mohanty and J.D. Axe, Phys. Rev. Lett 57, 1157 (1986); Z. Cheng, R. Savit and R. Merlin, Phys. Rev. B. 37, 4375 (1988).

[5] M.S. Vasconcelos and E.L. Albuquerque, Phys. Rev. B 57, 2826 (1998).

[6] M. Quilichini and T. Janssen, Rev. Mod. Phys. 69, 277 (1997).

[7] P.M.C. de Oliveira, E.L. de Albuquerque and A.M. Mariz, Physica A 227, 206 (1996).

[8] C.G. Bezerra and E.L. Albuquerque, Physica A 245, 379 (1997); 255, 285 (1998).

[9] D.H.A.L. Anselmo, M.G. Cottam and E.L. Albuquerque, J. Phys.: Condens. Matter 12, 1041 (2000).

[10] C.G. Bezerra, J.M. de Araújo, C. Chesman and E.L. Albuquerque Phys. Rev. B 60, 9264 (1999).

[11] C. Tsallis, L.R. da Silva, R.S. Mendes, R.O. Vallejos and A.M. Mariz, Phys. Rev. E 56, R4922 (1997).

[12] R.O. Vallejos, R.S. Mendes, L.R. da Silva and C. Tsallis, Phys. Rev. E 58, 1346 (1998). 
[13] R.O. Vallejos and C. Anteneodo, Phys. Rev. E 58, 4134 (1998).

[14] P. Carpena, A.V. Coronado and P. Bernaola-Galván, Phys. Rev. E 61, 2281 (2000).

[15] E.M.F. Curado and M.A. Rego-Monteiro, Phys. Rev. E 61, 6255 (2000).

[16] R.E. Camley, J. Mag. Mag. Mat. 200, 583 (1999).

[17] I.K. Schuler, S. Kim and C. Leighton, J. Mag. Mag. Mat. 200, 571 (1999).

[18] R. Merlin, J.P. Valladares, A. Pinczuk, A.C. Gossard and J.H. English, Solid State Commun. 84, 87 (1992).

[19] E.L. Albuquerque, Solid State Commun. 99, 311 (1996).

[20] M.G. Cottam and D.J. Lockwood: in Light Scattering in Magnetic Solids, John Wiley, 1986.

\section{Figure Captions}

1. The spin wave spectra for the Fibonacci structure. Here the in-plane wavevector is taken to be equal to 0.2 .

2. Specific heat versus temperature for the Fibonacci structure. We have plotted the specific heat profiles up to the $9 t h$ generation number. The inset shows the low temperature behavior of the specific heat.

3. Log-log plot of the specific heat versus temperature for the generation numbers of the Fibonacci quasiperiodic sequence. Observe a different behavior for the even $(n=4,6,8$, and 10) and odd $(n=5,7$, and 9$)$ generation numbers.

4. $C(T)$ vs $\log (T)$ plot to show the log-periodicity effects in the Fibonacci structure. 


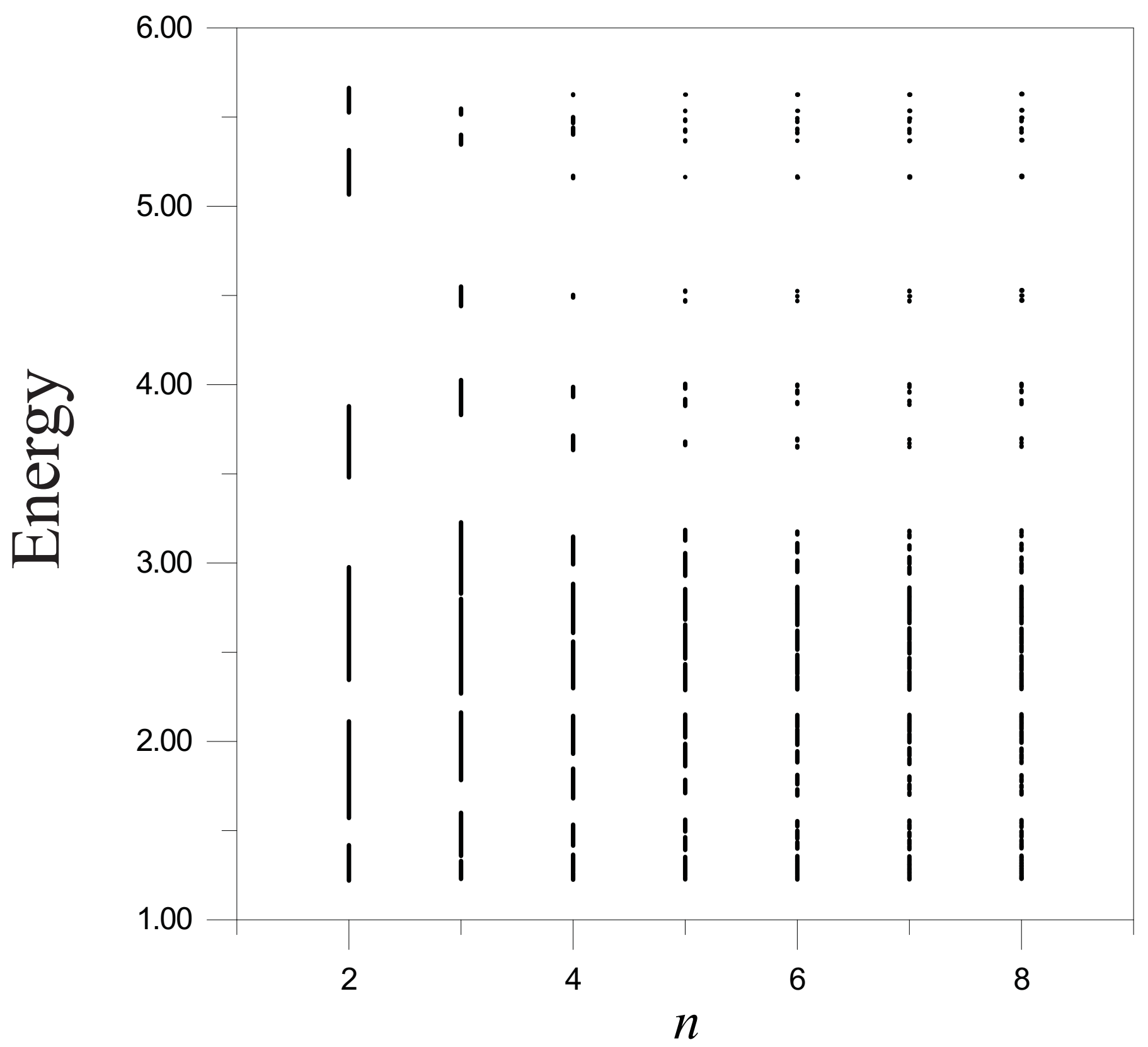




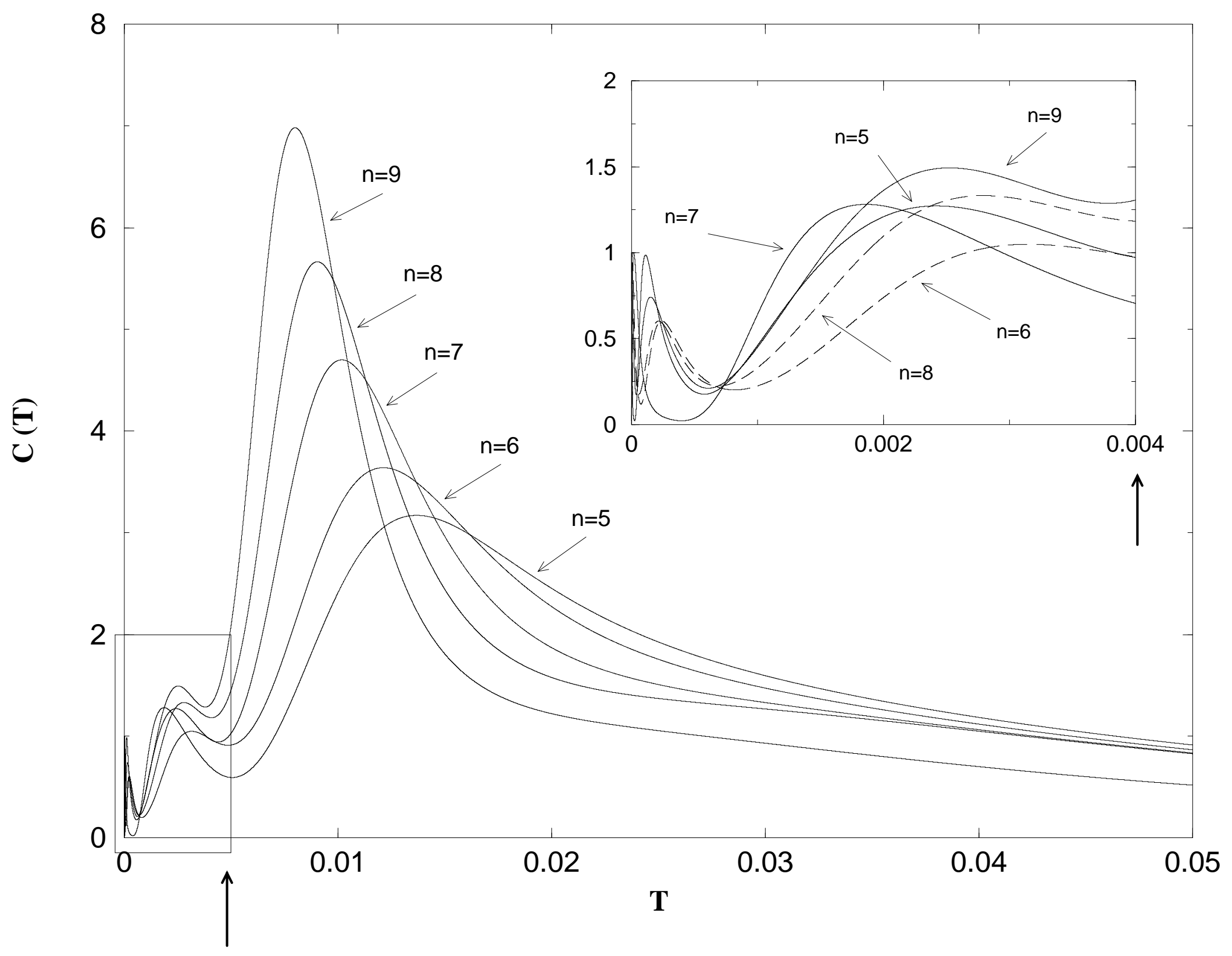




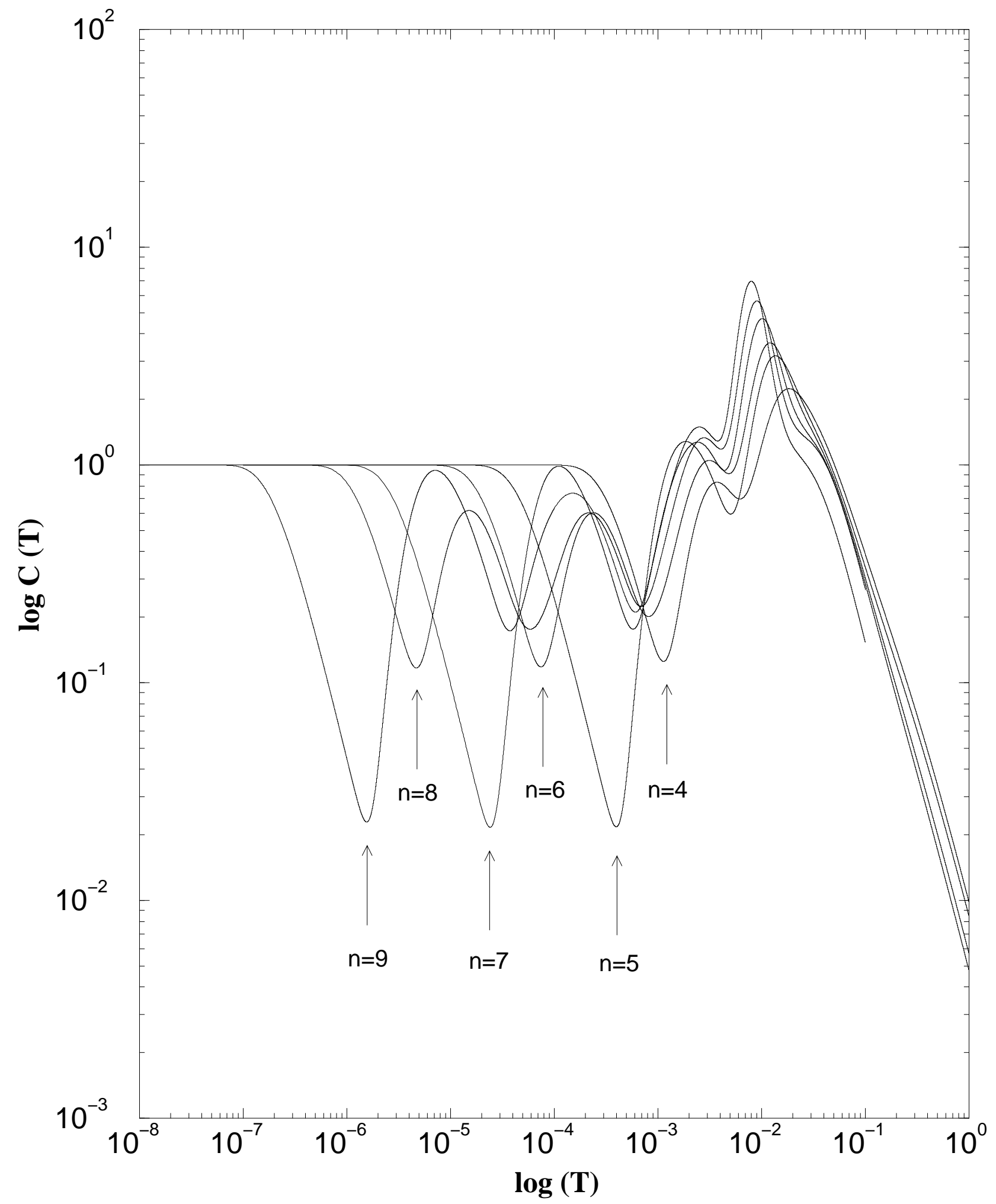




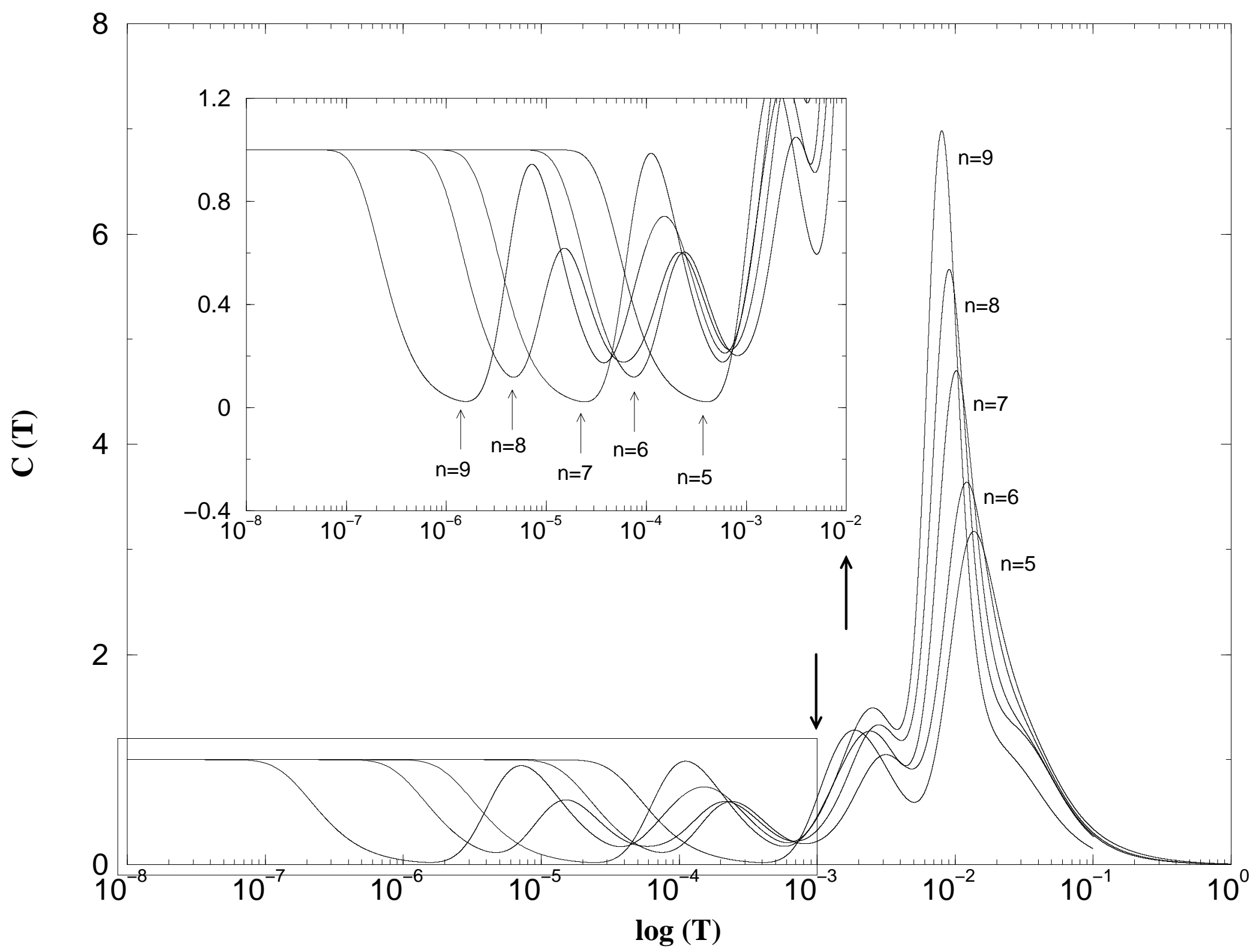

